\title{
MUPBED - Interworking Challenges in a Multi- Domain and Multi-Technology Network Environment
}

\author{
Hans-Martin Foisel ${ }^{1}$, Jan Spaeth ${ }^{2}$, Carlo Cavazzoni ${ }^{3}$, \\ Henrik Wessing ${ }^{4}$, Mikhail Popov ${ }^{5}$ \\ ${ }^{1}$ Hans-Martin Foisel, T-Systems/Deutsche Telekom, Goslarer Ufer 35, D-10589 Berlin, \\ Germany, Hans-Martin.Foisel@t-systems.com \\ ${ }^{2}$ Jan Späth, Ericsson, Gerberstr. 33, 71522 Backnang, Germany \\ ${ }^{3}$ Carlo Cavazzoni, Telecom Italia, Via G. Reiss Romoli 274, I-10148 Torino, Italy \\ ${ }^{4}$ Henrik Wessing, Technical University of Denmark, Bldg. 343, Oersteds Plads, 2800 \\ Kgs. Lyngby, Denmark \\ ${ }^{5}$ Mikhail Popov, Acreo AB, Electrum 239, SE-164 40 Kista, Sweden
}

\begin{abstract}
Today's data transport networks are evolving continuously towards customer oriented and application aware networks. This evolution happens in Europe in a highly diverse network environment, covering multiple network domains, layers, technologies, control and management approaches. In this paper, the issues, challenges and the solutions developed in the IST project MUPBED (,Multi-Partner European Test Beds for Research Networking“; www.ist-mupbed.eu) for seamless interworking in a typical European heterogeneous network environment are described, addressing horizontal, interdomain, and vertical, inter-layer topics related to data plane, control plane and applications.
\end{abstract}

Keywords: ASON/GMPLS, Test Network, Multi-Domain, Multi-Layer, Interworking, Intelligent Optical Networks

\section{Introduction}

Seamless interworking in a heterogeneous and fast evolving multi-domain, multilayer, multi-technology network environment is one of the top challenges network operators are facing today, indifferent of their background, e.g. incumbent, newcomer, local or global player, commercial or research network service provider. Additionally, the customer and application awareness of the networks have to be increased, the network flexibility enhanced leading to provisioning of so-called "OnDemand Network Services". This network scenario fits very well to the current European network environment and will most likely also be applicable to the foreseeable future.

Solutions to cope with these network environments are based on the data plane level on standards of ITU-T (International Telecommunication Union - Telecommunication Standardization Sector) and IE and Electronics 
Engineers - Standards Association) and on the control plane level on ASON/GMPLS (Automatically Switched Optical Network, Generalised Multi-Protocol Label Switching) standards of ITU-T, IETF (Internet Engineering Task Force) and Implementation Agreements of the OIF (Optical Internetworking Forum). Several ASON/GMPLS test network activities were carried out worldwide [1 - 8] aiming at gaining first experiences from these new network functions and providing valuable feedback to the standardisation bodies and forums.

The IST-Project MUPBED, "Multi-Partner European Test Bed for Research Networking" $[9,10]$ investigates and evaluates many of these interworking issues conceptually and experimentally. The practical evaluations have been carried out in a European scale multi-domain, multi-layer ASON/GMPLS test network, in a joint effort by network operators, industry partners, NRENs (National Research and Education Networks) and research institutes/universities. The test network includes multiple domains, each with a set of different technologies, e.g. IP/MPLS, Ethernet, $\mathrm{SDH}$, all optical cross connects and different control and management approaches.

This paper reports on interworking solutions developed in the MUPBED project, applicable to these heterogeneous network environments on the European as well as on the global scale.

The paper is structured in 4 sections. In section II the European network environment is described. Section III and IV cover the data and control plane interworking solutions, which have been developed, implemented and evaluated in the MUPBED test network.

\section{European Networks - a Heterogeneous Environment}

Networks have been portioned, layered or deployed with different technologies because of many reasons related to, e.g. investments, operation, services, or they simply have become heterogeneous because of acquisitions. Fig. 1 depicts schematically such a wide variety of different network domains with respect to the data plane technology implemented, whereas Fig. 2 illustrates the mix of different solutions at the control plane level. These scenarios are applicable for an intra- as well as inter-carrier network environment. Nevertheless, seamless interworking among all these different approaches is strongly needed and highly desirable for future-proof flexible and efficient solutions. Mandatory to achieve this goal is the integration of data and control plane functions to provide powerful new network functionalities. This integration results in challenges regarding to horizontal interworking, e.g. among network domains and to vertical interworking, e.g. among the different network layers and applications. These twofold interworking directions are schematically depicted in Fig. 3. Most of them were tackled and solved in the MUPBED test network, which is based on a similar divergent set of implementations at the five local test beds at TI/Torino, TID/Madrid, Acreo/Stockholm, PSNC/Poznan, and DT/Berlin, and at the European research network's implementation of their interconnections. 


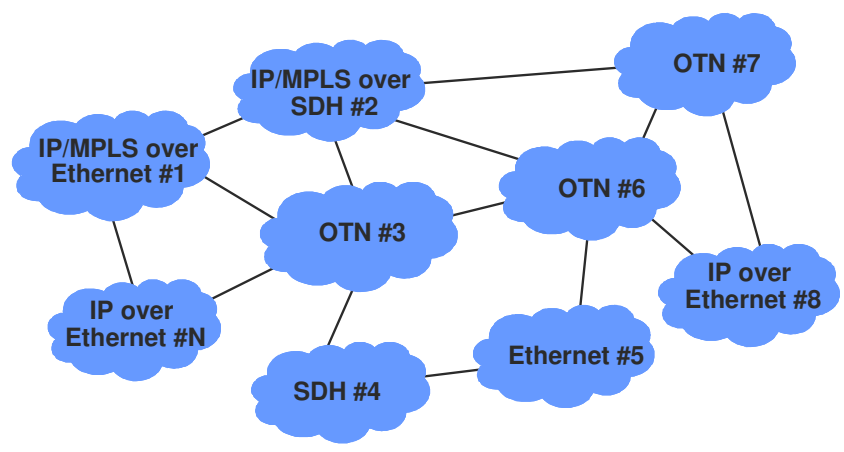

Fig. 1. Data plane: Multi-domain, multi-layer, multi-technology network scenario

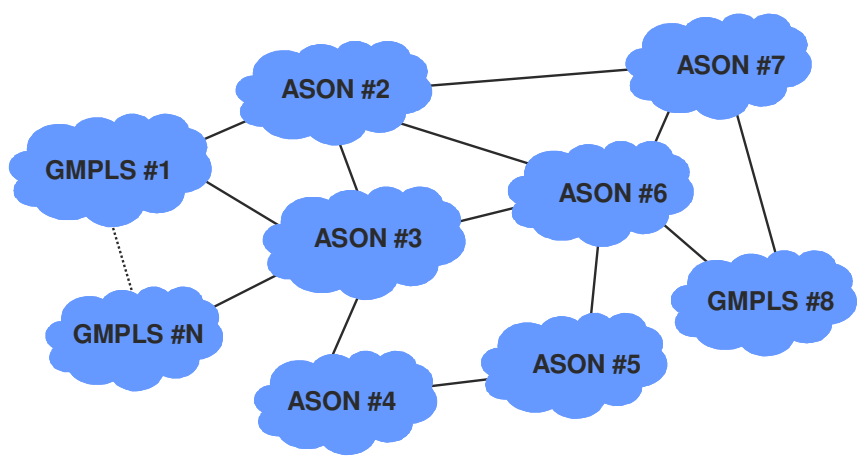

Fig. 2. Control plane: Multi-domain ASON/GMPLS network scenario

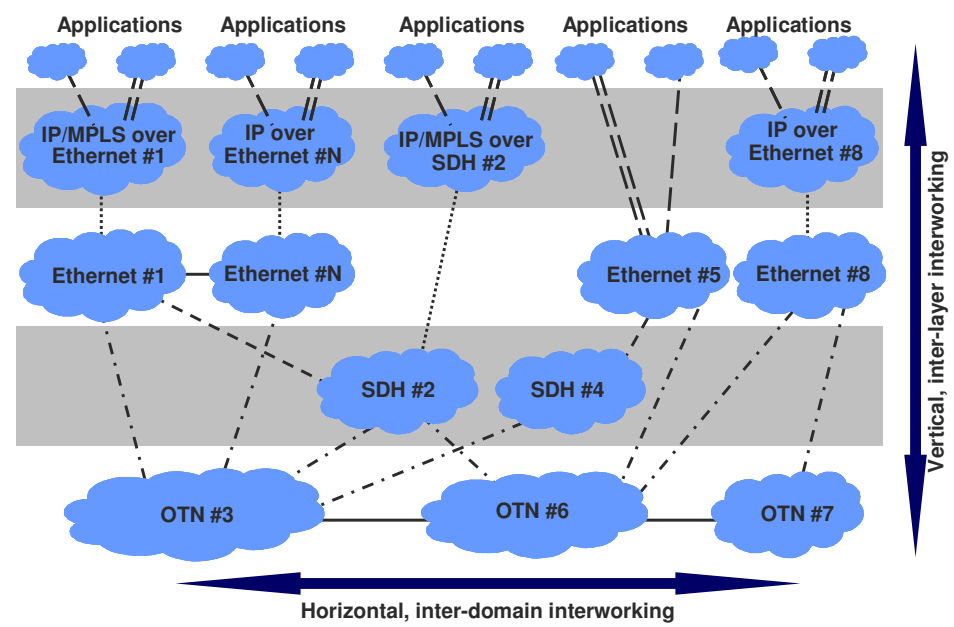

Fig. 3. Horizontal and vertical interworking areas in a multi-domain, multi-layer, multitechnology network environment, most of them tackled and solved in the MUPBED test network 
The European research network situation is a good example for a heterogeneous multi-carrier network scenario, with multiple National Research and Education Networks (NRENs) and GÉANT2 (the European research backbone network). The European research networks are organised and structured as follows: Campus networks and selected national and European projects are interconnected to the respective NREN, which enables and supports nationwide interconnectivity. These national research networks are currently based on individual (and therefore largely different) network architectures, technologies, functions, vendor equipment and network control/management implementations. It is most likely that this heterogeneity and individuality per domain will be maintained in the foreseeable future. Additionally to their national coverage, the NRENs provide interconnectivity to the European research backbone network GÉANT2 enabling a European scale connectivity and, additionally, long haul connections to non-European research networks. To enable such pan-European interconnections, GÉANT2 has to interoperate with all the individual NREN networks and is always involved in a multidomain network scenario, including mainly the connection configuration and control. Today, in most of the NRENs, the interworking is realised at the IP layer only. However, in the future it can be expected that interworking is required for various other network layers and technologies as well, to efficiently support large bandwidth and high QoS services.

Within the MUPBED test network the heterogeneity of the implementation per domain or test bed has been maintained. No alignment of these different data, control and management plane solutions has been postulated, but standard-based solutions have been required at the inter-domain interfaces, to enable seamless interworking within the MUPBED network as well as with other European and national project's test beds and even on a global scale at the OIF Worldwide Interoperability Demonstration 2005.

\section{Data Plane Interworking Areas in the MUPBED Test Network}

It is worth mentioning that even data plane interworking of different multi-layer networks might raise several challenges, although standards are available. This interworking is especially challenging because the involved network domains are generally based on different technologies, network platforms, vendor equipment and operational processes. Table 1 gives an overview of potential mapping procedures and solutions among network layers, highlighting the manifold solutions and options available. The solutions marked in grey in this table show implementations in the MUPBED test network.

In MUPBED, a very first step has been to provide connectivity among the local test beds based on transparent data links. With today's technologies, this could be best achieved with SDH/OTN interconnections between the five MUPBED test bed sites. However, the MUPBED project had to find a solution, which is supported at the interdomain interfaces by all local test beds and the European research networks. This 
results into a provisioned - statically configured - full-mesh topology of "transparent Layer 2" inter-connections, based on Ethernet over IP/MPLS transport (Fig. 4). It shows the five MUPBED test beds, the different technologies they are based on, reflecting very well the divergent European network environment, as well as the involved NRENs and GÉANT2 networks. All switching functions of the MUPBED network were allocated within the different MUPBED test bed sites. In such a way a multi-domain network in a truly multi-partner environment with participants from industry and research community has been setup.

Table 1. Data plane standard based mapping solution overview in multi-domain, multi-layer, multi-technology networks; marked options were implemented in the MUPBED test network

\begin{tabular}{|c|c|c|c|c|}
\hline $\begin{array}{c}\text { NIM } \\
(\mathbf{M} \text { over } \mathbf{N})\end{array}$ & IP/MPLS & Ethernet & SDH & OTN \\
\hline IP/MPLS & $\begin{array}{l}\text { LSPx - LSPy } \\
\text { interworking }\end{array}$ & EoMPLS or L2TP & & \\
\hline Ethernet & $\begin{array}{c}\text { LSP } \leftrightarrow \text { VLAN } \\
\text { mapping or plain } \\
\text { transport }\end{array}$ & $\begin{array}{c}\text { VLANx - VLANy } \\
\text { interworking or } \\
\text { stitching }\end{array}$ & $\begin{array}{c}\text { Emulation } \\
\text { (restricted } \\
\text { usability) }\end{array}$ & \\
\hline SDH & GFP/VCAT/LCAS & GFP/VCAT/LCAS & Basic feature & \\
\hline OTN & GFP/VCAT/LCAS & GFP/VCAT/LCAS & $\begin{array}{c}\text { Basic OTN } \\
\text { feature }\end{array}$ & Basic feature \\
\hline
\end{tabular}

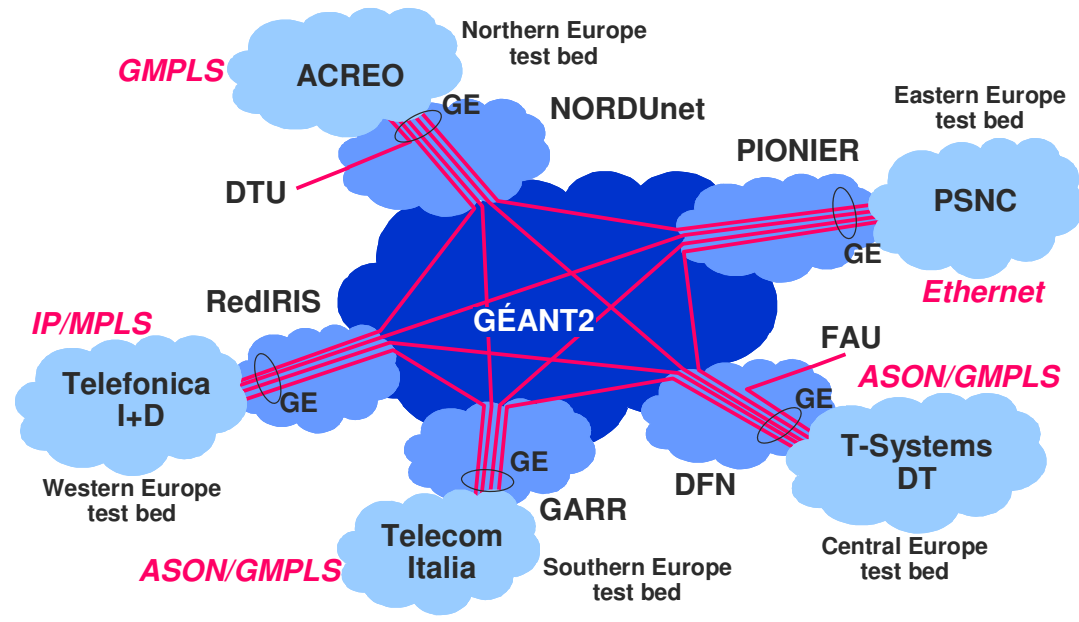

Fig. 4. Data plane implementation in the MUPBED test network - all five local test beds are interconnected via Ethernet over IP/MPLS links over the NREN and GÉANT2 networks

As an example how the end-to-end interworking of these different data plane implementations has been achieved within the MUPBED test network, the detailed implementation and interworking challenges and solutions at DT/Berlin are depicted 
in Fig. 5. The LSPs in the GÉANT2 IP/MPLS platform have been stitched to the LSPs in the IP/MPLS platform of the NRENs, here the X-WIN network of DFN. At the NRENs PoP at the location of the MUPBED test bed, these LSPs have been mapped into VLANs. In DT's local test bed, an Ethernet metro network performs the VLAN resolution and translation functions and makes those VLANs available on physically separated Gigabit-Ethernet (GE) ports, which in turn are connected to GE ports of the Ericsson NG-SDH domain. By setting up Ethernet Soft-PermanentConnections (Eth-SPCs) with a variable bandwidth between VC-4 to VC-4-7v, these ports can be interconnected to any desired other MUPBED test bed or application location. All these inter-domain and inter-layer mapping schemes are standard-based and interoperable solutions, resulting in the highest possible degree of interworking capabilities.

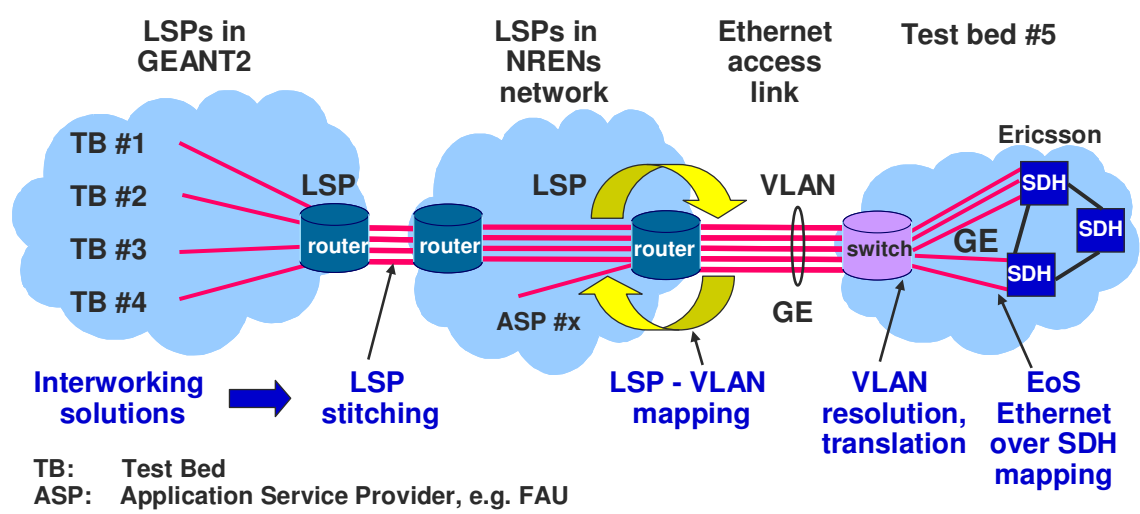

Fig. 5. Data plane interworking solutions in the MUPBED test network - Allocation of functions among GÉANT2, NREN networks and MUPBED local test beds

\section{Control Plane Interworking Areas in the MUPBED Test Net-work}

Interworking at the control plane level is even more challenging than at the data plane, because the standards and specifications are still in progress, therefore early prototype implementations need to be chosen to accomplish an automatic and seamless multi-domain and multi-layer interworking in the MUPBED test network.

The ASON/GMPLS inter-domain implementations (Fig. 6) have been following a network architecture which is suitable to the current - and most likely also for the near future European networks - very heterogeneous network environment, with many separated network domains largely consisting of different technologies, network architecture, and operational mechanisms. Therefore, solutions for seamless (automatic) inter-working among these network domains are a key issue to be solved for the next generation of research networks. The expected result is an increase in the multi-domain connection dynamics, the possibility for on-demand customer-oriented services, and a significant reduction of manual configurations. 
This network architecture and the concept to maintain the individual architecture and technology approaches in each of the five MUPBED test beds while enabling automatic interworking among the domains has been applied to the MUPBED network scenario, resulting into a network topology as depicted in Fig. 7. Furthermore, this concept allows using OIF inter-domain interfaces, the interoperable implementations of which were proven and demonstrated at the OIF Worldwide Interoperability Demonstration 2005 [8].

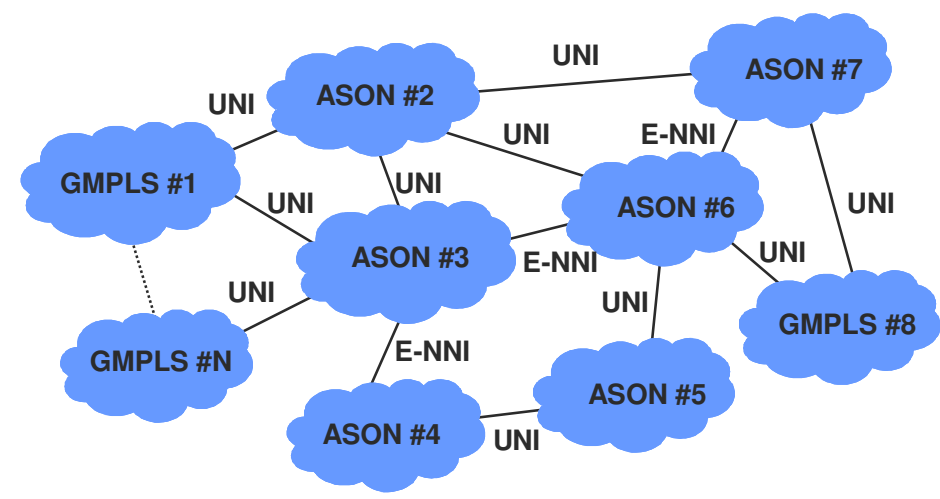

Fig. 6. Control plane: ASON/GMPLS multi-domain and multi-layer solution for a heterogeneous multi-domain network, using OIF inter-domain interfaces.

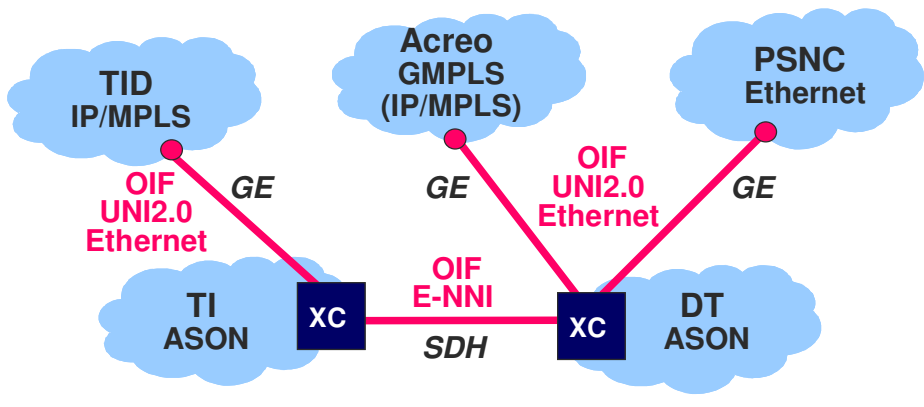

UNI-C 2.0 Ethernet proxy server implementations

Fig. 7. Control plane: ASON/GMPLS network architecture implementation in the MUPBED test network

Additionally to the interworking of network domains and layers, the MUPBED project has addressed the application-network interface issues, aiming to make the MUPBED ASON/GMPLS test network application-aware. As depicted in Fig. 8, a chain of functions has been chosen to interface the applications with the OIF UNI-C (client side), comprising Application Programming Interfaces (API), the adaptation function and finally the ASON/GMPLS control plane-aware UNI-C.

The main objective of the API is to provide a uniform access to the adaptation function with a simple communication for the applications, completely decoupled from any ASON/GMPLS signaling or knowledge of the underlying transport 
technology. The API implementation chosen in MUPBED is based on Web Service technologies, which enables easy communication on port 80 (HTTP), thus avoiding most of the firewall problems. The functional range of the API comprises the following three main communication messages: resource request, resource release and status request.

The Adaptation Function (AF) is introduced as responsible for interfacing with the network control plane and for deciding, when new network resources should be allocated. The adaptation function receives resource requests from the application via the API, and is responsible for translating and triggering these requests to the network. In this way, decoupling between applications and the currently used transport network technology is ensured.

The adaptation function controls the establishment of connections by interfacing to the UNI-C and the information available at this interface. Therefore, the adaptation function does not consider the network topology of the ASON/GMPLS layer, as it is simply aware of the edge-to-edge connections that are associated with the UNI-C it controls.

The OIF UNI specifies the signaling messages between the adaptation function and the network control plane. At the adaptation function side a UNI-C proxy server or RSVP agent provides the needed control plane functions.

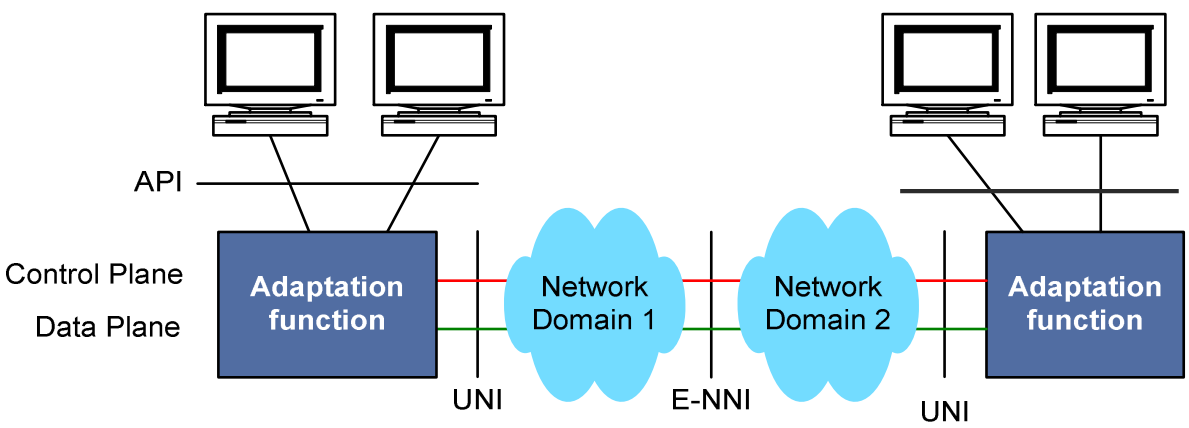

Fig. 8. Vertical and horizontal interworking: application-network interworking principle via API - Adaptation module - OIF UNI and E-NNI functions

In the MUPBED project, the following three application-network interface solutions have been developed (Fig. 9) and implemented [9]:

- The Network Provider Stack implementation implements an advanced resource allocation scheme in a multi-layer IP/MPLS over ASON/GMPLS network. As test application it uses a storage and backup software

- The Standalone or GUI implementation includes the resource allocation and is not integrated directly with specific applications. This makes it suitable as a separate, manually controlled tool for applications where integration of these functions could not be easily made

- In the Socket Stack Solution the applications do not communicate through an API and adaptation function to the UNI-C interface. Instead they directly execute socket calls in the UNI-C proxy server 


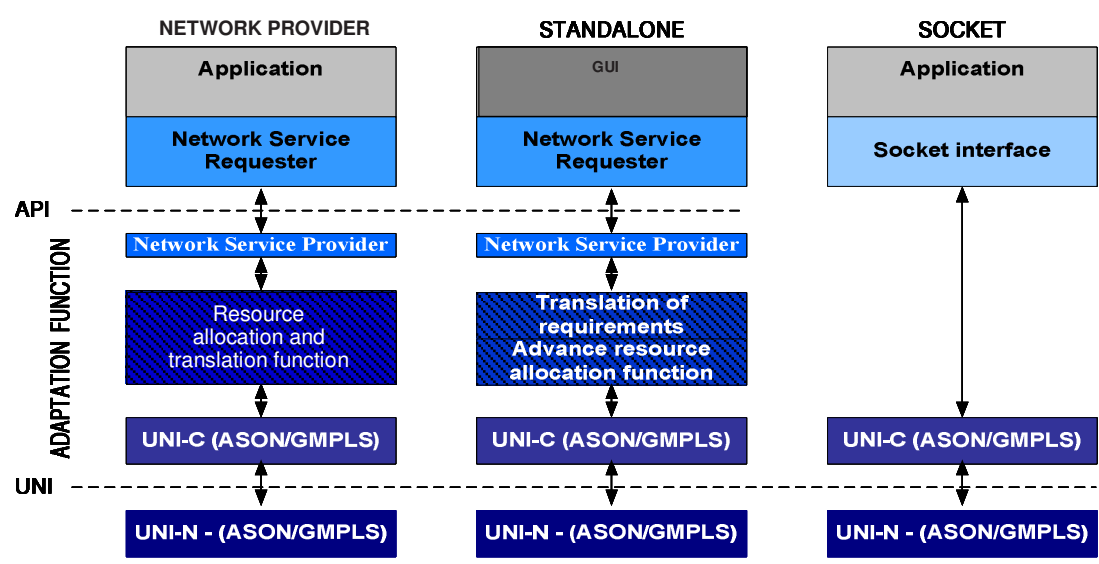

Fig. 9. Application-network interworking solutions implemented in the MUPBED project

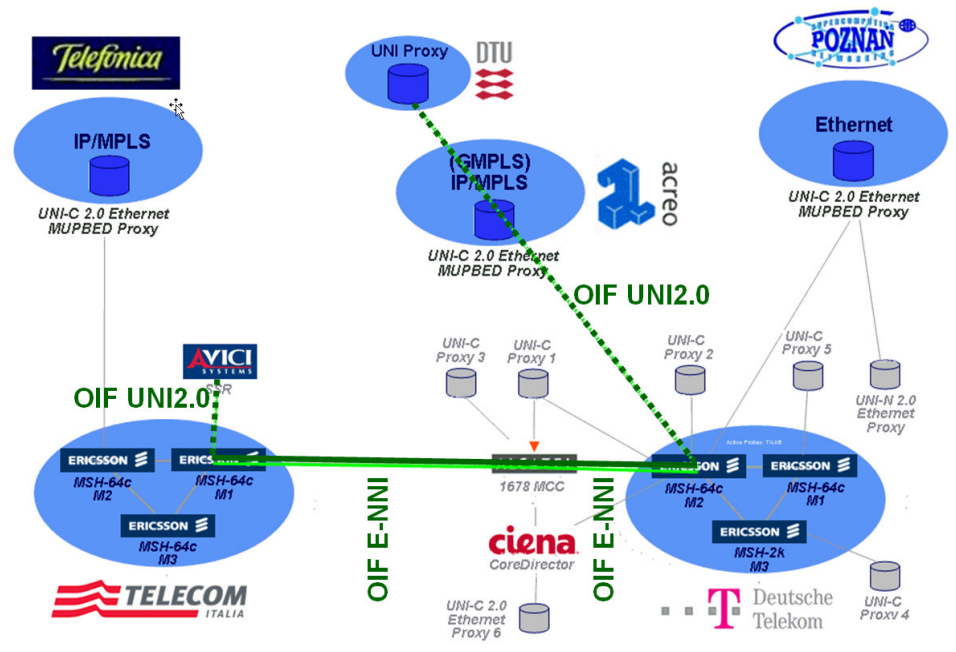

Fig. 10. Control plane: Example of the application-network interworking based on Standalone Application Vertical Integration Stack, depicting the topology of an Ethernet switched connection DTU/Copenhagen - DT/Berlin - TI/Torino initiated at DTU

The most flexible standalone or GUI implementation has been implemented, tested and demonstrated in the MUPBED test network. Fig. 10 shows a screen shot of the MUPBED network control plane level topology display, highlighting the Ethernet switched connection setup from DTU/Copenhagen via multiple ASON/GMPLS network domains at DT and TI to the UNI-C instance (Avici router) at TI, clearly illustrating the smooth interworking capabilities of applications with transport and data network domains and elements. 


\section{Conclusions}

The MUPBED project has implemented and tested solutions for seamless interworking in a heterogeneous multi-domain and multi-layer network environment, on the data and control plane level, suitable for the current and foreseeable future network environment of the European research networks and carriers. These solutions enable on-demand transport services based on standard-compliant ASON/GMPLS control plane technologies, ensuring the highest possible level of interoperability.

Additionally, seamless interworking with a wide variety of Ethernet-based clients has been considered in the project, with a special emphasis on highly-demanding applications, which could interwork with ASON/GMPLS networks via API and adaptation functions.

Acknowledgments. The work reported in this paper has been partly supported by the European Commission within the FP-6 project MUPBED (Multi-Partner European Test Beds for Research Networking) under contract number IST-511780.

The responsibility for the content of this paper is with the authors. The authors thank all colleagues from the MUPBED project for their work, which has been the basis for this paper.

\section{References}

1. C. Cavazzoni, R. Morro, R. Muñoz, R. Martínez, F. Galán, S. Szuppa, H. M. Foisel, H. Dentler, M. Herpers, J. Liebenow, Juan P. Fdez-Palacios, S. Spadaro, J. Comellas, "NOBEL phase 2 - Large scale ASON/GMPLS network demonstrator", ECOC2006 Workshop on Workshop on ASON/GMPLS Implementations in Field Trials and Carrier Networks, www.ist-mupbed.eu

2. PHOSPHORUS overview presentation, http://www.ist-phosphorus.eu/documents.php

3. P. Kaufmann, "VIOLA-Testbed: Current State and First Results", TERENA Networking Conference Proceedings, June 2006, Catania

4. T. Lehman, J. Sobieski, B. Jabbari, „DRAGON: A Framework for Service Provisioning in Heterogeneous Grid Networks", IEEE Communications Magazine, March 2006, Volume 44 , No 3

5. HOPI white paper: http://networks.internet2.edu/hopi/hopi-documentation.html

6. Cheetah: X. Zhu, X. Zheng, M. Veeraraghavan Z. Li, Q. Song, I. Habib N. S. V. Rao, "Implementation of a GMPLS-based Network with End Host Initiated Signaling", IEEE ICC 2006 conference proceedings, paper OS11-1

7. Y. Sameshima, S. Okamoto, W. Imajuku, T. Otani, Y. Okano, "JGN II Testbed Demonstration of GMPLS Inter-Carrier Network Control with Actual Operational Consideration, ECOC 2006 Proceedings, paper We 4.1.5

8. J.D. Jones, L. Ong, M. Lazer, Interoperability update - dynamic Ethernet services via intelligent optical networks, IEEE Optical Communication, Nov 2005, Vol3, No4, pp. 2-11

9. MUPBED project web page, presentations and Deliverables

10. C. Gerlach, H.-M. Foisel, S. Szuppa, A. Weber, „MUPBED: A multi-domain ASON/GMPLS test network“, NOC 2006 Proceedings, pp. 395-402 